Original Article

\title{
Accuracy of a Digital Weight Scale Relative to the Nintendo Wii in Measuring Limb Load Asymmetry
}

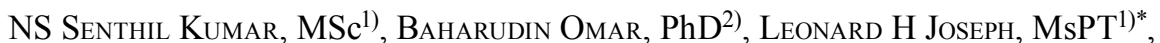 \\ Nor Hamdan MY, MD ${ }^{3)}$, Ohnmar Htwe, MD ${ }^{3)}$, Nursalbiyah Hamidun, Bsc, PT ${ }^{1}$ ' \\ 1) Physiotherapy Program, School of Rehabilitation Sciences, Faculty of Health Sciences, Universiti \\ Kebangsaan Malaysia: 5th Floor, Bangunan Yayasan Selangor, Jalan Raja Muda Abdul Aziz 50300, \\ Kuala Lumpur, Malaysia \\ 2) Department of Biomedical Sciences, Faculty of Health Sciences, Universiti Kebangsaan Malaysia, \\ Malaysia \\ 3) Department of Orthopedic and Traumatology, Faculty of Medicine, Universiti Kebangsaan Malaysia \\ Medical Centre, Malaysia
}

\begin{abstract}
Purpose] The aim of the present study was to investigate the accuracy of a digital weight scale relative to the Wii in limb loading measurement during static standing. [Methods] This was a cross-sectional study conducted at a public university teaching hospital. The sample consisted of 24 participants $(12$ with osteoarthritis and 12 healthy) recruited through convenient sampling. Limb loading measurements were obtained using a digital weight scale and the Nintendo Wii in static standing with three trials under an eyes-open condition. The limb load asymmetry was computed as the symmetry index. [Results] The accuracy of measurement with the digital weight scale relative to the Nintendo Wii was analyzed using the receiver operating characteristic (ROC) curve and Kolmogorov-Smirnov test (K-S test). The area under the ROC curve was found to be 0.67 . Logistic regression confirmed the validity of digital weight scale relative to the Nintendo Wii. The D statistics value from the K-S test was found to be 0.16 , which confirmed that there was no significant difference in measurement between the equipment. [Conclusion] The digital weight scale is an accurate tool for measuring limb load asymmetry. The low price, easy availability, and maneuverability make it a good potential tool in clinical settings for measuring limb load asymmetry.

Key words: Accuracy, Digital weight scale, Nintendo Wii Fit
\end{abstract}

(This article was submitted Jan. 14, 2014, and was accepted Feb. 16, 2014)

\section{INTRODUCTION}

Limb load asymmetry (LLA) is common in clinical conditions related to the lower limbs such as degenerative joint disease, soft-tissue injuries, joint replacements, amputations, and stroke ${ }^{1,2)}$. LLA is defined as an uneven distribution of body weight on one lower extremity when compared with the other extremity ${ }^{2}$. LLA may occur due to pain, reduced muscle strength, and/or impaired joint mobility $^{1)}$. Eventually, LLA may cause degeneration of the joint and osteopenia ${ }^{1)}$. Hence, LLA may be an important clinical variable for investigation of impairments and the need for further rehabilitation.

A variety of equipment is available to measure LLA, including biofeedback systems, ambulatory devices with sensors, the Tekscan MatScan system, and force platforms ${ }^{3)}$.

\footnotetext{
*Corresponding author. Leonard H Joseph (E-mail: leonardjoseph85@hotmail.com)

C 2014 The Society of Physical Therapy Science. Published by IPEC Inc. This is an open-access article distributed under the terms of the Creative Commons Attribution Non-Commercial No Derivatives (by-ncnd) License $<$ http://creativecommons.org/licenses/by-nc-nd/3.0/>
}

These pieces of equipment provide better accuracy and reliable LLA measurements. However, they are rarely available in practice due to their high costs and are confined to laboratory settings. In addition, these pieces of equipment also require ample space and specialized training to handle them in clinical settings.

The aforementioned limitations of these pieces of equipment highlight the need for a simple, inexpensive device to measure LLA in daily practice. Recent studies have utilized a visual simulation gaming tool, such as the Nintendo Wii (Wii), to measure LLA ${ }^{3,4)}$. The Wii provides reliable and valid LLA measurements when compared with the gold standard force platform $(\text { ICC }=0.77-0.89)^{5}$. Nevertheless, the Wii requires a television for data capture, and it is less mobile. Recent literature has proposed measurement of LLA using 2 pieces of digital weight scale (DWS) equipment ${ }^{4}$. The DWS is inexpensive, portable, and easy to utilize in the clinical setting ${ }^{3,4)}$. However, the accuracy of LLA measurement obtained from the DWS is uncertain. Accuracy refers to how close a value is to the accepted val$\mathrm{ue}^{5)}$. It is indicated as the measurement closeness to truth. In the present study, accuracy refers to the closeness of agreement between the limb loading measurement values from the DWS to the measurement values from the Wii. Hence, 
this study focuses on the accuracy of the DWS by comparison with the Wii.

\section{SUBJECTS AND METHODS}

This experimental study was conducted in the human movements and health research unit of a public university teaching hospital. The study samples comprised 12 osteoarthritis Kellgren- Lawrence grade 2 and 3 and 12 healthy participants. Participants were recruited if they could understand commands and stand independently and were without any neurological disorders. Ethical approval was obtained from the university research committee. Prior to data collection, informed consent was obtained from all participants. LLA was measured using two Beurer DWSs (BEU-GS27-0007, Beurer, Ulm, Germany), each with a precision of $0.01 \mathrm{~kg}$. The maximum measurement capability of each DWS was $150 \mathrm{~kg}$. Prior to data collection, the accuracy of the two DWSs was tested with dead weights to ensure zero errors ${ }^{2}$. The two DWSs were placed on flat, hard flooring at a distance equivalent to the participant's shoulder width ${ }^{6}$. Participants were instructed to stand in a relaxed posture while facing forward with their bare feet centrally placed on each DWS ${ }^{7}$. The readings were noted after standing on the scales for 10 seconds. Three trials of measurement were noted and averaged for data analysis.

The Nintendo Wii Fit system (Nintendo of America Inc., Redmond, WA, USA) consists of a balance board, console, remote, and television for data acquisition. The Wii console was used to synchronize the data from the balance board to a television. The measurement procedure used for the DWS was repeated to collect data from the Wii. The participants were given 5 minutes of rest between DWS and Wii measurements. The sequences of equipments were randomized. One researcher collected the data throughout the study. The loading measurements from both lower limbs were computed for LLA analysis using the symmetry index (SI) ${ }^{8}$. The SI is derived from a mathematical formula that provides LLA values in percentages.

$$
\text { SI }=\frac{\operatorname{Load}_{\text {Right }}-\operatorname{Load}_{\text {Left }}}{0.5\left(\operatorname{Load}_{\text {Right }}+\operatorname{Load}_{\text {Left }}\right)} \times 100 \%
$$

Accuracy was analyzed using the Statistical Package for the Social Sciences (version 20). A receiver operating characteristic (ROC) curve was utilized to compare the measurement data obtained from the Wii and DWS. Twosample Kolmogorov-Smirnov (K-S) tests were utilized in conjunction with the ROC, as this test is sensitive to differences in measurement values, for both the location and shape of the empirical cumulative distribution functions of the DWS and Wii.

\section{RESULTS}

The study group consisted of 24 participants, 12 osteoarthritis (OA) participants ( 8 female, 4 male) and 12 healthy participants ( 8 female, 4 male). The mean age of the osteoarthritis participants was $61.25 \pm 6.68 \mathrm{yrs}$, mean body mass
Table 1. Comparison of SI values from the DWS and Wii using the two-sample Kolmogorov-Smirnov (K-S) test

\begin{tabular}{lcccc}
\hline & Mean \pm SD & $95 \%$ CI & High & Low \\
\hline SI (DWS) & $4.16 \pm 2.7$ & $3.02-5.30$ & 0.10 & 1.33 \\
SI (Wii) & $4.16 \pm 2.9$ & $2.93-5.39$ & 0.11 & 2.38 \\
\hline
\end{tabular}

index (BMI) was $27.26 \pm 3.36 \mathrm{~kg} / \mathrm{m}^{2}$ and mean Visual Analogue Scale (VAS) score was $4.83 \pm 1.59$. The mean age of the healthy participants was $61.83 \pm 7.86 \mathrm{yrs}$, and their mean BMI was $26.71 \pm 4.76 \mathrm{~kg} / \mathrm{m}^{2}$. Based on the VAS scores, none of the healthy participants reported pain in the lower limbs.

The ROC curve was used to test the accuracy of the DWS relative to the Wii. The area under the curve was 0.67 with a $95 \%$ confidence interval $(p=0.06)$. The upper bound and lower bound values were 0.50 and 0.82 respectively. The standard error of measurement was $0.08 \%$ of change between the symmetry indexes of the DWS and Wii. The $\mathrm{K}-\mathrm{S}$ test results are shown in Table 1.

\section{DISCUSSION}

In practice, existence of asymmetry in lower limb loading among healthy populations is a controversial issue. Measurement of limb loading during while standing is crucial in various orthopedic and neurological conditions ${ }^{1,8,9)}$. However, the current measurement tools are not readily available in clinical practice. Hence, the present study proposed the use of two DWSs for measurement of LLA and tested the accuracy relative to the Nintendo Wii.

The results of the present study demonstrate the measurement accuracy of the DWS relative to the Wii. The area under the ROC curve was found to be 0.65 , which is between 0.5 to 1.0 , indicating a best fit between the two pieces of equipment. In addition, the logistic regression line indicates that the results are not by chance. Hence, the DWS and Wii produce similar LLA measurements during a standing task. According to the K-S test, the maximum difference between the cumulative distributions of the SI obtained from the DWS and Wii is given by a D value of 0.16 , which is greater than the critical value $(\mathrm{p}=0.86)$. This proves that the symmetry index scores obtained from the DWS and Wii are comparable.

The study findings could be ascribed to the technical configurations of the two pieces of equipment, as the DWS and Nintendo Wii balance board uses strain gauge sensors to sense load shifts ${ }^{5}$. They could also be attributed to the calibration and zero error checking conducted for the two DWSs prior to data collection. The two DWSs used in this study had a precision of $0.01 \mathrm{~kg}$, which indicates that the closeness of agreement between independent measurements might have reduced the chance of uncertainty. Also, this study used three trails, and the measurements were averaged for computation, which might have reduced the random error, as supported by the central limit theorem ${ }^{10)}$. Finally, the standard procedure and instructions given to the participants during the measurements might have been responsible for the similarities in measurements between the 
devices. The DWS could fill the gap with regard to tools for measurement of LLA based on its advantages in terms of portability, ease of operation, instant readings, and proven accuracy. The findings of the present study could indicate opportunities for clinicians and researchers to decide on the usefulness of the DWS as a clinical and research tool for measurement of LLA in the field of rehabilitation.

\section{REFERENCES}

1) Christiansen CL, Stevens-Lapsley JE: Weight-bearing asymmetry in relation to measures of impairment and functional mobility for people with knee osteoarthritis. Arch Phys Med Rehabil, 2010, 91: 1524-1528. [Medline] [CrossRef]

2) Adegoke BO, Olaniyi O, Akosile CO: Weight bearing asymmetry and functional ambulation performance in stroke survivors. Glob J Health Sci, 2012, 4: 87-94. [Medline]

3) Hurkmans HL, Bussmann JB, Benda E, et al.: Techniques for measuring weight bearing during standing and walking. Clin Biomech (Bristol,
Avon), 2003, 18: 576-589. [Medline] [CrossRef]

4) Bohannon RW, Larkin PA: Lower extremity weight bearing under various standing conditions in independently ambulatory patients with hemiparesis. Phys Ther, 1985, 65: 1323-1325. [Medline]

5) Clark RA, Bryant AL, Pua Y, et al.: Validity and reliability of the Nintendo Wii Balance Board for assessment of standing balance. Gait Posture, 2010, 31: 307-310. [Medline] [CrossRef]

6) Caldwell C, MacDonald D, MacNeil K, et al.: Symmetry of weight distribution in normals and stroke patients using digital weigh scales. Physiother Theory Pract, 1986, 2: 109-116. [CrossRef]

7) Pereira LC, Botelho AC, Martins EF: Relationships between body symmetry during weight-bearing and functional reach among chronic hemiparetic patients. Rev Bras Fisioter, 2010, 14: 229-266. [Medline]

8) Robinson RO, Herzog W, Nigg BM: Use of force platform variables to quantify the effects of chiropractic manipulation on gait symmetry. J Manipulative Physiol Ther, 1987, 10: 172-176. [Medline]

9) Agrawal V, Gailey R, Gaunaurd I, et al.: Weight distribution symmetry during the sit-to-stand movement of unilateral transtibial amputees. Ergonomics, 2011, 54: 656-664. [Medline] [CrossRef]

10) Rice A: John: Mathematical Statistics and Data Analysis. Pacific Grove: Duxbury Press, 1995, p 53 